\title{
Consideraciones prácticas sobre la elaboración del portafolios del estudiante: su aplicación al aprendizaje de la pronunciación del inglés ${ }^{1}$
}

\author{
Julio ROCA DE LARIOS \\ Universidad de Murcia \\ Departamento de Didáctica de la Lengua y la Literatura \\ jrl@um.es
}

Sonia LÓPEZ SERRANO

Universidad de Murcia

Departamento de Filología Inglesa

lopezserrano.sonia@gmail.com

Recibido: septiembre 2010

Aceptado: marzo 2011

\section{RESUMEN}

En este trabajo presentamos una serie de consideraciones prácticas sobre la elaboración y el empleo del portafolios del estudiante como alternativa metodológica en la enseñanza universitaria. Delimitamos, en primer lugar, lo que se entiende por portafolios y señalamos las principales ventajas e inconvenientes que se le atribuyen. Describimos a continuación las etapas que se aconseja cubrir a la hora de introducir el portafolios del alumno como innovación metodológica, e ilustraremos cada una de estas fases con algunos ejemplos extraídos de un proyecto en el que se utilizó el portafolios para la enseñanza de la pronunciación del inglés en la universidad de Murcia. En una sección posterior, damos cuenta de los resultados de ese proyecto a partir de un breve análisis del contenido de los portafolios producidos por los alumnos y de sus percepciones a la hora de elaborarlos. Completamos el trabajo con un conjunto de recomendaciones relativas a la implantación del portafolios del alumno como recurso didáctico en la universidad.

Palabras clave: Portafolios, etapas de elaboración, repuesta de los alumnos, pronunciación del inglés.

Considérations pratiques sur l'élaboration du portfolio de l'étudiant : son application à l'apprentissage de la prononciation de l'anglais

\section{RÉSUMÉ}

Dans ce travail, nous présentons une série de considérations pratiques sur l'élaboration et l'utilisation du portfolio de l'étudiant comme alternative méthodologique dans l'enseignement

${ }^{1}$ El presente trabajo forma parte de un proyecto de investigación, financiado por el Ministerio de Ciencia e Innovación (FF12009-14155), titulado "Estrategias de uso en la adquisición formal del inglés como L2: uso, entrenamiento y resultados de aprendizaje". 
universitaire. En premier lieu, nous délimitons ce qu'on entend par portfolio, puis nous signalons les principaux avantages et inconvenients qui lui sont attribués. Ensuite, nous décrivons les étapes qu'il est conseillé de suivre lors de l'introduction du portolio de l'étudiant comme innovation méthodoloqique, et après cela nous illustrerons chacune de ces phases à l'aide de quelques exemples tirés d'un projet dans lequel le portfolio a été utilisé pour l'enseignement de la prononciation de l'anglais à l'université de Murcia. Nous continuons en rendant compte des résultats de ce projet à partir d'une brève analyse du contenu des portfolios produits par les étudiants ainsi que de leurs perceptions pendant le processus d'élaboration de ceux-ci. Finalement, nous complétons notre travail par une série de recommendations en relation avec l'implantation à l'université du portfoloio de l'étudiant comme recours didactique.

Mots-clés : Portolio, étapes d'élaboration, réponse des élèves, prononciation de l'anglais.

\title{
Some practical considerations on students' portfolios and its application in teaching and learning English pronunciation
}

\begin{abstract}
The present paper is intended as an empirically motivated discussion of some practical considerations on the use of students' portfolios as a methodological alternative to current university pedagogical practices. After describing the defining characteristics of the portfolio as well as its purported advantages and drawbacks, the main stages involved in its elaboration and use are discussed and illustrated with examples from a portfolio-based project aimed to teach English pronunciation at the University of Murcia. A brief analysis is then presented of the content of the portfolios produced by the students taking part in that project, followed by a summary of their perceptions on the elaboration process. Finally, a number of pedagogical recommendations are suggested as to the use of students' portfolios for syllabus planning and implementation.
\end{abstract}

Keywords: Portfolio, elaboration stages, student's perceptions, English pronunciation.

SUMARIO: 1. Introducción. 2. Definición de portafolios. 3. Supuestas ventajas e inconvenientes del portafolios. 4. Fases de elaboración del portafolios. 4.1. Fase preliminar. Presentación de los objetivos, contenido y organización del portafolios. 4.2. Fase de presentación y discusión de los criterios de evaluación. 4.3. Fase de recopilación y selección de evidencias. 4.4. Fase de reflexión sobre las evidencias. 5. Análisis del contenido de los portafolios de fonética. 5.1. Evidencias seleccionadas. 5.2. Criterios de selección utilizados. 5.3. La autoevaluación. 5.4. La percepción de los alumnos sobre la elaboración de los portafolios. 6. Conclusiones. Referencias bibliográficas.

\section{INTRODUCCIÓN}

Algunas de las propuestas emanadas del Espacio Europeo de Educación Superior se pueden interpretar, posiblemente, como un intento de cubrir las necesidades actuales de formación integral de los alumnos. Si tradicionalmente los contenidos impartidos en la enseñanza universitaria se referían, en el caso de muchas materias, a un conocimiento de carácter estático y enciclopédico que debía asimilarse casi 
exclusivamente de forma teórica y repetitiva, las nuevas propuestas abogan por incorporar a los planes de estudio conocimientos plurales y diversos, de naturaleza condicional, e integrados entre sí de forma compleja (Sharp, 1997). Sin embargo, es necesario reconocer que se ha prestado muy poca atención en nuestro medio al desarrollo sistemático y evaluación de las habilidades implicadas en la adquisición de ese nuevo tipo de conocimientos (observación, decodificación, análisis, síntesis, discriminación, valoración, argumentación, composición, etc.).

La puesta en práctica de procesos de enseñanza y aprendizaje que promuevan el desarrollo este tipo de habilidades entre los alumnos aparece, por tanto, como una tarea prioritaria. Esta tarea requerirá, por una parte, la ampliación del repertorio de metodologías docentes utilizadas hasta la fecha en la Universidad y, por otra, y en clara correspondencia con lo anterior, la construcción de un marco conceptual y de unos procedimientos de evaluación que ayuden tanto en las tareas de control y acreditación de los estudios cursados como en el análisis y comprensión del proceso de aprendizaje de los estudiantes. No en vano, muchas experiencias pretendidamente innovadoras pierden fuerza entre los alumnos cuando, al final del proceso, la comprobación de todos sus esfuerzos se reduce a la realización de una prueba o examen.

Se hace necesaria, por tanto, la búsqueda y realización de innovaciones didácticas centradas en la utilización de recursos que impulsen el desarrollo de habilidades complejas por parte de los alumnos y que, al mismo tiempo, faciliten la recogida de la información necesaria para llevar a cabo el análisis y la valoración del proceso. Entre la innovaciones que se vienen proponiendo para este fin destaca el empleo del portafolios, un recurso didáctico que, procedente del mundo del arte y de la arquitectura, ha sido trasplantado al mundo de la educación. En este trasplante, como sugieren Pozo y García (2006), el portafolios ha perdido algunas de sus características iniciales, pasando de ser un "contenedor rígido y cerrado" a convertirse en un "contenedor dinámico" que incluye un conjunto de documentos destinados a reflejar el rendimiento y la capacidad de reflexión de la persona que lo construye, de acuerdo con una serie de criterios previamente estipulados.

En el ámbito de la educación se suelen distinguir dos tipos de portafolios: el portafolios del docente, que sirve para reflejar el proceso de planificación, ejecución y evaluación de la docencia por parte del profesor y para reflexionar sobre el mismo (cf. Fernández March, 2004; Lyons, 1999), y el portafolios del estudiante, al que nos vamos a referir en las páginas que siguen. Antes de ello, sin embargo, conviene señalar que, debido a los procesos de auto-evaluación reflexiva subyacentes en ambos, el portafolios docente y el del estudiante son, en realidad, "las dos caras del mismo proceso de reflexión e innovación que tiene lugar en el aula" (Wright y otros, 1999, 90).

En este contexto, hemos centrado el presente trabajo en la elaboración y utilización del portafolios o carpeta del estudiante a través de la presentación de un conjunto de ideas prácticas ilustradas con algunos datos extraídos de una experiencia en la que se ha aplicado esta metodología a la docencia de la asignatura de Fonética Inglesa en el segundo curso de Magisterio de Lenguas Extranjeras, en la Facultad de 
Educación de la Universidad de Murcia. No se pretende aquí, por tanto, dar cuenta de las razones de tipo teórico, o incluso ideológico, que subyacen a la introducción del portafolios, cuestión para la que remitimos al lector a la literatura pertinente (cf. Arteaga y Fernández, 2000; Brown y Glasner, 2003; Bordas y Cabrera, 2001; Klenowski, 2005; Lyons, 1999).

Desde esta perspectiva, eminentemente práctica, delimitaremos, en primer lugar, lo que se entiende por portafolios y señalaremos las principales ventajas e inconvenientes que se le atribuyen. Describiremos a continuación las etapas o fases que se aconseja cubrir a la hora de introducir el portafolios del alumno como una innovación metodológica, e ilustraremos cada una de estas fases con algunos de los documentos utilizados en su elaboración y desarrollo durante la impartición de la asignatura anteriormente señalada. En una sección posterior, daremos cuenta de los resultados de ese proceso de elaboración a partir de un breve análisis del contenido de los portafolios producidos así como de la respuesta de los estudiantes ante su realización $\mathrm{y}$, finalmente, completaremos el trabajo con un conjunto de recomendaciones relativas a la implantación del portafolios del alumno como recurso didáctico.

\section{DEFINICIÓN DE PORTAFOLIOS}

Seguramente hay casi tantas definiciones del portafolios como autores se han ocupado del tema (Barberá, 2005). Hemos seleccionado tres de ellas:

- "Un portafolio didáctico es la historia documental estructurada de un conjunto (cuidadosamente seleccionado) de desempeños que ha recibido preparación o tutoría, y adoptan la forma de muestras de trabajo de un estudiante que sólo alcanzan realización plena en la escritura reflexiva, la deliberación y la conversación" (Shulman, 1999, en Lyons, 1999, 18).

- "El portafolio es una recopilación de evidencias (documentos diversos, viñetas, artículos de prensa, publicidad, páginas web, notas de campo, diarios, relatos....) consideradas de interés para ser guardadas por los significados con ellas construidos" (Agra, Gewerc y Montero, 2003).

- "El portafolio es un método de enseñanza, aprendizaje y evaluación que consiste en la aportación de producciones de diferente índole por parte del estudiante a través de las cuales se pueden juzgar sus capacidades en el marco de una disciplina o materia de estudio. Estas producciones informan del proceso personal seguido por el estudiante, permitiéndole a él y a los demás ver sus esfuerzos y logros, en relación a los objetivos de aprendizaje y criterios de evaluación establecidos previamente" (Ficha metodológica de la Universidad Miguel Hernández, Elche, 2006).

Si se aglutinan los diversos aspectos mencionados en esas definiciones, una caracterización global implicaría que el portafolios del estudiante, para ser considerado como tal, ha de rebasar la mera colección o recopilación de trabajos y concebirse como un instrumento de carácter longitudinal, con un contenido diversificado, que promueva la reflexión del estudiante sobre su proceso de 
aprendizaje y suponga diversos grados de colaboración entre los alumnos y el profesor. En efecto, se trata de un instrumento caracterizado por la flexibilidad y el dinamismo, que se presta a ser utilizado en una gran diversidad de situaciones de enseñanza y aprendizaje, con relación a una amplia gama de temas y para una amplia gama de propósitos. Además, al tener una orientación tanto hacia fuera (los materiales realizados y recopilados) como hacia adentro (significados albergados en su construcción), a su capacidad de almacenaje de productos, el portafolios añade la posibilidad continua de evidenciar cómo se van produciendo los procesos de enseñanza y aprendizaje desde el punto de vista de los protagonistas, por medio de la conversación reflexiva con los materiales de la situación (Schön, 1992). Finalmente, el portafolios supone una apuesta por la conjunción de la evaluación formativa con la de carácter sumativo (análisis de esfuerzos y logros con relación a los criterios de evaluación establecidos previamente). De hecho, para muchos autores la principal utilidad de este instrumento reside en las posibilidades que ofrece al profesor de llevar a cabo una evaluación auténtica del aprendizaje desde la perspectiva múltiple que surge a través de las evidencias seleccionadas y de la reflexión del alumno sobre las mismas, algo que, en principio, no cabe esperar de otros instrumentos de evaluación más estandarizados, a los que se supone una orientación más fragmentada (Arteaga y Fernández, 2000).

Cuatro son, por tanto, los aspectos principales que caracterizan al portafolios (Agra y otros, 2003):

- Reflejar la evolución y el logro del estudiante en su proceso de aprendizaje.

- Estimular la experimentación, la reflexión y la investigación.

- Potenciar el diálogo en torno a los problemas, los logros, los temas...los momentos claves del proceso.

- Reflejar el punto de vista personal de los protagonistas y favorecer su colaboración.

\section{SUPUESTAS VENTAJAS E INCONVENIENTES DEL PORTAFOLIOS}

Reconocer, sin más, el potencial del portafolios como un instrumento de innovación educativa, pasando por alto sus inconvenientes, constituiría un flaco favor a su expansión y a su correcto desarrollo. Se hace necesario, por tanto, tener también en cuenta sus posibles puntos débiles, algo que, además de ser éticamente exigible, puede ayudarnos a sopesar los supuestos beneficios didácticos que con cierta frecuencia se predican de su utilización. El listado que ofrecemos a continuación, en forma de tabla de doble entrada, es un resumen de las ideas que hemos hallado en la literatura sobre el tema: 
Tabla 1. Ventajas e inconvenientes potenciales del portafolios.

\begin{tabular}{|c|c|}
\hline Ventajas & Inconvenientes \\
\hline $\begin{array}{l}\text { Ofrece amplia información sobre el proceso } \\
\text { de aprendizaje y los logros del alumno a lo } \\
\text { largo del tiempo. }\end{array}$ & $\begin{array}{l}\text { No se dispone de un volumen suficiente de } \\
\text { investigación empírica sobre su eficacia. }\end{array}$ \\
\hline $\begin{array}{l}\text { Fomenta la autonomía, al permitir, en la } \\
\text { medida de lo posible, que el estudiante } \\
\text { decida aquellos aspectos en los que } \\
\text { personalmente desea profundizar. }\end{array}$ & $\begin{array}{l}\text { Los alumnos pueden sentir confusión y cierta } \\
\text { falta de claridad sobre los objetivos a } \\
\text { conseguir. }\end{array}$ \\
\hline $\begin{array}{l}\text { El estudiante tiene la oportunidad de } \\
\text { presentar evidencias sobre sus logros con } \\
\text { relación a la diversidad de aprendizajes que } \\
\text { ha interiorizado, los problemas que ha } \\
\text { experimentado y las estrategias que ha } \\
\text { utilizado en la ejecución de las tareas. }\end{array}$ & $\begin{array}{l}\text { El estudiante puede carecer de las habilidades } \\
\text { necesarias para realizar la recopilación y la } \\
\text { selección de las evidencias pertinentes. }\end{array}$ \\
\hline $\begin{array}{l}\text { Tiene un gran componente motivador y de } \\
\text { estímulo para los estudiantes de un trabajo } \\
\text { continuado donde se pueden ir comprobando } \\
\text { con cierta rapidez la relación entre el } \\
\text { esfuerzo realizado y los resultados } \\
\text { conseguidos. }\end{array}$ & $\begin{array}{l}\text { Implica un alto nivel de autodisciplina y de } \\
\text { responsabilidad que no todos los estudiantes } \\
\text { están, en principio, dispuestos a asumir. } \\
\text { Como consecuencia, algunos de ellos pueden } \\
\text { adoptar una actitud puramente formalista, o } \\
\text { incluso resistirse a realizarlo, cuando su } \\
\text { elaboración no representa un alto porcentaje } \\
\text { en la nota final. }\end{array}$ \\
\hline $\begin{array}{l}\text { La construcción del portafolios es una tarea } \\
\text { abierta (ill-defined) y compleja. Supone, por } \\
\text { tanto, la implicación cooperativa del profesor } \\
\text { y los estudiantes en su desarrollo. }\end{array}$ & $\begin{array}{l}\text { Al tratarse de una tarea compleja y abierta } \\
\text { (ill-defined), puede dar lugar a que no se } \\
\text { entienda el concepto en toda su profundidad. } \\
\text { Ello puede producir cierta inseguridad entre } \\
\text { los estudiantes, e incluso entre el mismo } \\
\text { profesorado. }\end{array}$ \\
\hline $\begin{array}{l}\text { Si se realiza en varias asignaturas, puede } \\
\text { favorecer el intercambio de puntos de vista y } \\
\text { la cooperación entre varios profesores. }\end{array}$ & $\begin{array}{l}\text { La utilización del portafolios significa un } \\
\text { cambio de estilo de enseñanza no siempre } \\
\text { fácil de asumir por los docentes. Posibles } \\
\text { causas: confianza en metodologías más } \\
\text { tradicionales, ratio de alumnos por aula, edad, } \\
\text { etc. }\end{array}$ \\
\hline $\begin{array}{l}\text { Proporciona al profesor la oportunidad de } \\
\text { evaluar de forma formativa y sumativa el } \\
\text { aprendizaje del alumno. }\end{array}$ & $\begin{array}{l}\text { En cuanto método de evaluación "auténtico", } \\
\text { presenta ciertos problemas: (i) el coste en } \\
\text { tiempo y en el número de personas que } \\
\text { pueden ser evaluadas simultáneamente; (ii) la }\end{array}$ \\
\hline
\end{tabular}




\begin{tabular}{|l|l|}
\hline & $\begin{array}{l}\text { complejidad y el carácter abierto de la tarea } \\
\text { puede dificultar el poder obtener una muestra } \\
\text { adecuada de la expresión de las habilidades a } \\
\text { evaluar; (iii) el efecto del contexto puede dar } \\
\text { lugar a que la evaluación sea excesivamente } \\
\text { subjetiva y tangencial. }\end{array}$ \\
\hline $\begin{array}{l}\text { El estudiante puede contar desde el principio } \\
\text { con los criterios con los que será evaluado su } \\
\text { trabajo, lo cual garantiza, sobre el papel, una } \\
\text { evaluación honrada. }\end{array}$ & $\begin{array}{l}\text { El estudiante puede mostrar indiferencia o } \\
\text { resistirse a los criterios que se le presentan. }\end{array}$ \\
\hline $\begin{array}{l}\text { El portafolios es un producto personalizado, } \\
\text { y, por tanto, puede reflejar con cierta } \\
\text { fidelidad la forma en que cada estudiante } \\
\text { aborda su propio proceso de aprendizaje. }\end{array}$ & $\begin{array}{l}\text { Suele darse cierta falta de fiabilidad en la } \\
\text { aplicación de los criterios de evaluación. } \\
\text { Asimismo, la evaluación de un documento } \\
\text { muy personal puede constituir una tarea } \\
\text { difícil de asumir en clases muy numerosas. }\end{array}$ \\
\hline $\begin{array}{l}\text { Es un instrumento que puede ayudar a que } \\
\text { los alumnos reflexionen sobre sus procesos } \\
\text { de aprendizaje a lo largo de periodos } \\
\text { extensos de tiempo. }\end{array}$ & $\begin{array}{l}\text { Si no seleccionan bien los aspectos claves o } \\
\text { no se establecen los mecanismos de control } \\
\text { adecuados, la misma complejidad de la tarea } \\
\text { puede conducir al alumno a un empleo de } \\
\text { tiempo excesivo en la realización del } \\
\text { portafolios, en detrimento del que debería } \\
\text { dedicar a otras materias. }\end{array}$ \\
\hline
\end{tabular}

\section{FASES DE ELABORACIÓN DEL PORTAFOLIOS}

Antes de decidirse a emplear el portafolio del alumno es aconsejable valorar los posibles beneficios que este recurso didáctico puede aportar en el contexto específico donde se ejerza la actividad docente. Si la decisión sobre su utilización es positiva, conviene entonces tener en cuenta un conjunto de aspectos a partir de los cuales se pueden ir concretando las características que tendrá el instrumento (Wolf y Dietz, 1998). Siguiendo a Barberá (2005), estos aspectos se refieren básicamente a:

- La autoría y la audiencia del portafolios (¿quién será evaluado y quién será el evaluador?, ¿será la evaluación compartida entre los estudiantes y el profesor, o será éste quien tenga la última palabra?).

- Los contenidos a desarrollar (¿qué objetivos y competencias servirán de hilo conductor?,¿qué conjunto de temas se desarrollarán?,¿en relación con qué contenidos del temario de la asignatura?).

- La estructura y organización concreta del portafolios (¿qué tipo de portafolios se tiene en mente -abierto, semi-estructurado, cerrado, formativo, sumativo?,¿qué apartados contendrá?). 
- Los criterios de evaluación (¿cómo se puede saber que un alumno ha aprendido?).

- El tipo de apoyo que se brindará a los alumnos (¿se desarrollarán tutorías especiales como preparación previa a la construcción del portafolios, o se seguirá un procedimiento de "esperar y ver qué pasa"?,¿qué tipo de revisiones se harán y por qué medios? ¿qué tipo de apoyo recibirán los estudiantes en sus procesos de reflexión?).

Una vez que se ha reflexionado sobre estos aspectos, la organización del proceso de enseñanza y aprendizaje centrado en el portafolios implica la adopción de una secuencia distribuida en fases interdependientes. Esta secuencia tiene principalmente un valor heurístico, que puede ser de gran ayuda para guiar al profesor y a los alumnos en la complejidad del proceso. De acuerdo con Barberá (2005) y Bermejo Bravo (2006), las fases por las que suele pasar la elaboración del portafolios son las que se presentan a continuación.

\subsection{Fase preliminar. Presentación de los objetivos, contenido y organización del portafolios.}

Este momento del proceso está destinado a dar a conocer a los estudiantes los puntos más importantes del proyecto y en qué medida les afectan e involucran. Un aspecto esencial en esta fase es hacerles conscientes de que la tarea a realizar no consiste simplemente en recopilar documentos de forma ordenada, sino que lo fundamental es el proceso de reflexión que se puede generar, a partir de ellos, en relación con los criterios estipulados. También es el momento de adoptar los primeros acuerdos con relación a la organización y distribución temporal de las diversas actividades y de dejar claro que, al tratarse de una tarea compleja, abierta y flexible, no se pueden delimitar todos sus aspectos desde el principio, por lo que los alumnos deben asumir que la construcción del portafolios estará sujeta a revisiones y adaptaciones progresivas.

Las instrucciones dadas a los estudiantes pueden reflejar estos elementos con distintos grados de precisión. El Ejemplo 1 (véase más abajo) ilustra las instrucciones que recibieron los estudiantes de Magisterio de $2^{\circ}$ curso de Inglés (Lenguas Extranjeras), a los que nos hemos aludido anteriormente, durante el primer cuatrimestre del curso 2005-06 para la construcción del portafolios en la asignatura de Fonética:

\section{Ejemplo 1}

El portafolios o carpeta es una selección de evidencias (entendiendo por tal, cualquier tipo de trabajo que lleves a cabo en casa o en clase) que habrá que entregar al finalizar el cuatrimestre. Dicha selección será revisada por el profesor los días 28 de Octubre y el 30 de Noviembre. Además de los trabajos seleccionados, en el portafolio también habrás de incluir los criterios que has utilizado para realizar esa selección y para juzgar 
sus méritos. La idea básica es que dicha selección constituya una representación clara y precisa de la trayectoria de tus esfuerzos y logros en las distintas áreas de la Fonética que estudiaremos, de forma que llegue a ser un verdadero instrumento de autoevaluación y mejora de tu propio proceso de aprendizaje.

Otro aspecto fundamental de esta fase es que las intenciones educativas queden plasmadas en un conjunto de objetivos o competencias que funcionen a modo de hilo conductor en la construcción del portafolios. Estos objetivos se pueden agrupar en bloques y sirven tanto de punto de partida para la recopilación y selección de evidencias como de piedra de toque para la reflexión sobre las mismas. Obviamente, no todas las competencias seleccionadas por el profesor pueden ser utilizadas desde el primer día por los estudiantes, sino que el empleo de unas u otras variará a lo largo del proceso y en clara conexión con el desarrollo del programa de la asignatura. En el ejemplo siguiente se presentan las competencias que, en el portafolios anteriormente citado, sirvieron de hilo conductor para la selección de evidencias y para la reflexión sobre las mismas por parte de los estudiantes:

\section{Ejemplo $2^{2}$}

1. Capacidad para distinguir la composición fonética de las palabras (estructura segmental, estructura silábica, secuencia acentual, etc.).

2. Capacidad para interiorizar y aplicar a su propia producción las reglas más comunes de acentuación de palabras.

3. Capacidad para utilizar las reglas de cambio de acento en las palabras debido a la inserción de sufijos.

4. Capacidad para concatenar secuencias desconocidas de sonidos.

5. Capacidad para manejar con soltura las pautas rítmicas propias de la lengua inglesa desde el punto de vista de la percepción y de la producción.

6. Capacidad para monitorizar y corregir las propias producciones orales y lecturas en voz alta en inglés con relación a todos los puntos anteriores.

\subsection{Fase de presentación y discusión de los criterios de evaluación}

Según distintos autores, la transparencia en la selección y presentación de los criterios mediante los cuales se llevará a cabo la evaluación es una de las grandes ventajas de este procedimiento metodológico, tanto más cuanto que la forma en que se evalúe condicionará la forma en que el estudiante abordará su labor de aprendizaje. De ahí la importancia de exponer con claridad a los alumnos cómo y en función de qué criterios serán evaluados antes de que aborden la elaboración del portafolios. De

${ }^{2}$ Nota: Sólo la competencia número 1 constituyó un objetivo al iniciarse la construcción del portafolios. Las competencias restantes se fueron incorporando a lo largo del cuatrimestre en correspondencia con el desarrollo del programa de la asignatura 
este modo, podrán dirigir sus esfuerzos a la recopilación de evidencias y al desarrollo de tareas que cumplan con los requisitos exigidos (Barberá, 2005).

Una de las competencias que los alumnos habían de desarrollar en el portafolio de Fonética era su capacidad para monitorizar y corregir sus propias producciones orales y de lectura en voz alta (véase Ejemplo 2 más arriba, competencia 6). De acuerdo con ello, se pedía a los alumnos que valorasen su propia pronunciación en cada una de las evidencias presentadas que tuviesen carácter productivo. Esta tarea de autoevaluación era posteriormente contrastada y valorada por el profesor. Tanto las autoevaluaciones de los estudiantes como las valoraciones posteriores del profesor se basaban en los criterios incluidos en el Ejemplo 3, que los alumnos recibieron antes de iniciar la construcción del portafolios:

\section{Ejemplo $3^{3}$}

1. El alumno es capaz de detectar en su propia producción errores de tipo segmental relativos a:

- La pronunciación de grupos de consonantes en posición inicial y final.

- La implosión de oclusivas en posición intermedia.

- La pronunciación de la schwa y la /ae/.

- La articulación de fricativas y africadas.

- Las terminaciones de las palabras según los sufijos que contienen.

- La articulación de diptongos y triptongos.

2. El alumno es capaz de detectar en su propia producción errores de tipo suprasegmental relativos a:

- La acentuación de formas fuertes.

- La pronunciación de las formas débiles.

- El ajuste entre palabras de sonidos consonánticos entre sí, de consonante y vocal y de vocales entre sí.

- La división de la cadena hablada en grupos de pensamiento.

- La pronunciación de los fenómenos de asimilación estudiados.

- La identificación de efectos de "pregnancia" de unos sonidos a otros en palabras próximas de la cadena hablada.

- La realización de pausas.

3. El alumno es capaz de describir los errores cometidos desde el punto de vista fonético y de aventurar una explicación.

\footnotetext{
${ }^{3}$ Nota: No se esperaba que los alumnos fuesen capaces de aplicar todos los criterios desde el primer día, sino sólo aquellos aplicables a los errores de tipo segmental (apartado 1). Los criterios relativos a los apartados 2 y 3 (errores suprasegmentales y explicaciones) habrían de ir aplicándolos a lo largo del cuatrimestre, según se tratasen en clase los fenómenos fonéticos a los que hacen referencia.
} 


\subsection{Fase de recopilación y selección de evidencias}

La recopilación y selección de las evidencias viene determinada por los objetivos y competencias que constituyen el hilo conductor del portafolios. Al no ser éste un inventario de todo el trabajo realizado, se trata de que el estudiante elija las actividades, documentos o artefactos más representativos de su aprendizaje. La selección y organización de los mismos deben poner de manifiesto ante el profesor y, en su caso, ante los compañeros, que es capaz de desarrollar, entre otras, sus habilidades de observación, decodificación, discriminación y valoración.

Aunque existen diversas clasificaciones de evidencias, aquí citaremos la utilizada por Collins (1991, en Bermejo Bravo, 2006), por considerarla bastante útil y completa. Este autor distingue cuatro tipos de evidencias: los artefactos, las reproducciones, los testimonios y las producciones. Los artefactos son documentos relativos a las actividades realizadas en clase y a los trabajos propuestos por el profesor (apuntes de clase, redacciones, exámenes, resúmenes de lecturas, etc.). Las reproducciones son los materiales recopilados fuera del aula que ilustran o ayudan a comprender determinados conceptos (entrevistas, vídeos, discusiones on-line, etc.). Los testimonios incluyen los documentos de revisión y opinión de las personas encargadas de la formación del alumno (notas de una entrevista con el profesor de una asignatura para revisar un trabajo, valoraciones de otros profesores, etc.). Finalmente, las producciones están relacionadas con los documentos preparados por el estudiante para proporcionar una estructura y un sentido global al portafolio; por lo general, se materializan en las explicaciones sobre los objetivos a conseguir, las reflexiones sobre su desarrollo y las justificaciones e informes que acompañan a las evidencias.

Es conveniente señalar, por último, que el grado de libertad del alumno a la hora de incluir un tipo u otro de evidencias estará en función del tipo de portafolios por el que se opte: libre, semi-estructurado o estructurado. El ejemplo que sigue a continuación muestra el grado de libertad que se dio a los alumnos a la hora de recopilar y seleccionar las evidencias para la construcción del portafolios de Fonética que venimos comentando:

\section{Ejemplo 4}

Teniendo en cuenta que esta asignatura es de carácter muy práctico, es de esperar que los trabajos incluidos en la carpeta reflejen tu evolución en la mejora de la pronunciación con relación a las competencias que se te han proporcionado (véase ejemplo 3). Para ello sería conveniente que, junto a una selección de actividades de trascripción, incluyeses una cinta de cassette o grabaciones en MP3 que contengan una selección de las actividades de audición, lecturas en voz alta y resto de actividades de pronunciación que vayas realizado a lo largo del cuatrimestre. 


\subsection{Fase de reflexión sobre las evidencias}

Ésta es posiblemente la fase crítica en la elaboración del portafolios. De hecho, casi todos los autores consultados coinciden en afirmar que lo que distingue al portafolios de una mera recopilación de documentos es la posibilidad que ofrece este instrumento de echar la vista atrás para reflexionar sobre los puntos fuertes y débiles del proceso y, desde esta perspectiva, poder configurar expectativas, estrategias y propósitos de mejora desde el punto de vista personal del aprendiz. Implica, por tanto, el despliegue de habilidades complejas de análisis y síntesis, y quizá sea por ello por lo que se suele considerar que esta fase es la que presenta mayores dificultades para los estudiantes (Smith y Tillema, 2003). De ahí la necesidad de proporcionales apoyos explícitos antes, durante y con posterioridad a sus procesos de reflexión, apoyos que tendrán un carácter más a menos dirigido según el tipo de portafolios que los alumnos hayan de construir.

En el caso del portafolios de Fonética, como puede verse a continuación, la ayuda proporcionada a los estudiantes se materializó en una escala que les permitiese analizar el grado de conexión entre las evidencias presentadas y la reflexión sobre las mismas a través de las competencias implicadas:

\section{Ejemplo 5}

Nivel 0: No se presenta ninguna evidencia.

Nivel 1: Las evidencias presentadas son incompletas o no están interpretadas.

Nivel 2: Las evidencias receptivas presentadas muestran cierto reconocimiento de la diversidad de aspectos segmentales y suprasegmentales presentes en ellas.

Las evidencias productivas presentadas muestran cierta habilidad emergente en la articulación de aspectos segmentales y suprasegmentales a nivel de palabra, oración y texto.

Nivel 3: Las evidencias receptivas presentadas muestran un nivel adecuado de reconocimiento de la diversidad de aspectos segmentales y suprasegmentales presentes en ellas.

Las evidencias productivas presentadas muestran una habilidad no siempre eficiente en la articulación de aspectos segmentales y suprasegmentales a nivel de palabra, oración y texto.

Nivel 4: Las evidencias receptivas presentadas muestran un nivel excelente de reconocimiento de la diversidad de aspectos segmentales y suprasegmentales presentes en ellas.

Las evidencias productivas presentadas muestran una habilidad claramente eficiente en la articulación de aspectos segmentales y suprasegmentales a nivel de palabra, oración y texto 
Además de la reflexión sobre las evidencias presentadas, algunos autores aconsejan que la construcción del portafolios finalice con la posibilidad de que los alumnos puedan plasmar en forma de texto estructurado y comprensible los elementos de las fases anteriores, de manera que queden claras al lector las conexiones internas y personales de los contenidos de aprendizaje. Se trataría de un momento en el que la impronta más personal del aprendiz ha de hacerse más visible, ya que, en principio, ni el progreso en el aprendizaje ni la forma de presentar dicho progreso son idénticos entre un estudiante y otro. En nuestro caso, sin embargo, las características de la asignatura de Fonética y la sobrecarga académica que estaban afrontando los alumnos durante el cuatrimestre desaconsejaron la posibilidad de esta reflexión final.

Por otra parte, la literatura sobre el tema aconseja que a lo largo de todas las fases de construcción del portafolios se lleven a cabo procesos de revisión y diálogo entre el profesor y los estudiantes, a fin de que éstos últimos puedan ir consolidando o reconduciendo su trabajo. Incluso se sugiere que la realización de estos procesos no tenga lugar a muy largo plazo, sino que el portafolios sea revisado con cierta frecuencia a lo largo del curso para que cumpla con sus objetivos formativos. Hay, por tanto, que concretar fechas de entrega y de revisión, así como especificar qué elementos van a ser objeto de evaluación en cada caso concreto. Por ello, es conveniente diseñar instrumentos (o hacer uso de instrumentos previamente diseñados) que faciliten y centren este tipo de comunicación entre los estudiantes y el profesor.

Algunos de los listados y escalas presentadas con anterioridad (véanse, por ejemplo, los ejemplos 2, 3 y 5) pueden utilizarse para estos fines, pues, como puede deducirse de su lectura, obligan al profesor a sistematizar sus valoraciones y a tratar los aspectos fuertes y débiles del trabajo de los alumnos y, a su vez, dan a éstos la oportunidad de incorporar en su portafolios las recomendaciones recibidas, sumándolas a sus propios comentarios y autovaloraciones.

Por lo que respecta a la asignatura de Fonética que estamos comentando, la importancia de las respuestas del profesor en la orientación que los alumnos dieron finalmente al portafolios queda demostrada en el ejemplo 6 (véase también la siguiente sección sobre el análisis de los contenidos).

\section{Ejemplo 6}

Como se especificaba en las instrucciones (véase ejemplo 1), los alumnos hicieron una primera entrega del portafolios a finales de Octubre, aproximadamente tres semanas después de iniciarse el curso. A la vista de los datos, difíciles de evaluar de forma fiable en bastantes casos, se especificaron aún más las instrucciones sobre el tipo de evidencias que debían seleccionar y sobre el tipo de actividades que debían realizar con ellas. Así, se estipuló que, a partir de ese momento, deberían preparar y grabar la lectura en voz alta de un conjunto de textos ingleses en dos momentos distintos, antes y después de ser tratados en clase. Deberían, además, auto-evaluar su pronunciación al realizar esas lecturas, tarea para la que se les facilitó un listado de aspectos en los que deberían fijar su atención (véase Ejemplo 3). 


\section{ANÁLISIS DEL CONTENIDO DE LOS PORTAFOLIOS DE FONÉTICA}

Una vez explicitados los principales pasos a tener en cuenta en la elaboración del portafolios como instrumento de enseñanza y aprendizaje, es hora de centrarse en la experiencia llevada a cabo. El análisis cualitativo del contenido de los portafolios de Fonética se llevó a cabo a través de tres apartados que se encuentran en clara consonancia con las instrucciones dadas a los alumnos para su elaboración (véase ejemplo 1): las evidencias seleccionadas, los criterios de selección utilizados, y las actividades de auto-evaluación llevadas a cabo. Antes de exponer los resultados de este análisis, hay que tener en cuenta que, como se sugiere en el ejemplo anterior, la elaboración del portafolios de Fonética tuvo dos etapas claramente diferenciadas: una primera etapa, desde el comienzo del curso hasta finales de Octubre (fecha de la primera entrega), y una segunda, desde principios de Noviembre hasta el final del cuatrimestre (fecha de la segunda entrega).

\subsection{Evidencias seleccionadas}

El análisis de los portafolios refleja que, en la primera etapa, la gama de evidencias seleccionadas resultó más variada que en la segunda etapa. No en vano, al iniciarse el cuatrimestre, las instrucciones proporcionadas a los alumnos les daban un amplio margen de maniobra para llevar a cabo sus propios procesos de recopilación. Ello dio como resultado una gran diversidad de actividades en las evidencias seleccionadas: análisis de canciones en busca de fonemas inexistentes en español, lectura de extractos del material cubierto hasta ese momento en clase o de novelas simplificadas, repetición de sonidos problemáticos, realización de trascripciones de palabras polisílabas y de frases que las incluyeran, y descripciones de los procedimientos seguidos para preparar la lectura de frases y textos. Hay que señalar que la descripción de estas actividades podían llegar a ser muy minuciosas, incluyendo, en bastantes ocasiones, la división del texto en grupos semánticos, el empleo de flechas para señalar la entonación requerida, la trascripción y la pronunciación repetida de frases y palabras por separado en razón de su dificultad, o el intento de integración de todos estos elementos en cuantas lecturas globales del texto fueran precisas.

Debido a las dificultades implicadas en la evaluación de ese conjunto tan dispar de evidencias, el profesor de la asignatura, como se ha puesto de manifiesto anteriormente (véase ejemplo 6), modificó las instrucciones para la elaboración del portafolios de cara a la segunda entrega, y esta decisión condicionó el carácter más uniforme del tipo de evidencias seleccionadas por los alumnos a partir de ese momento. En efecto, un análisis de los portafolios elaborados en ese periodo refleja que, en la mayor parte de los casos, el trabajo de los alumnos se centró de forma casi exclusiva, según se aconsejaba en las instrucciones, en la preparación, análisis y lectura en voz alta (con el apoyo, a veces, de trascripciones) de trozos de novelas simplificadas, cuentos para niños y textos expositivos. 


\subsection{Criterios de selección utilizados}

La especificación de los criterios utilizados para la selección de las evidencias y de las tareas realizadas aparece en 34 de los 45 portafolios entregados en Octubre (primera entrega). Esta proporción decrece, sin embargo, en los portafolios presentados al final del cuatrimestre (12 de 45) debido, sin duda, al hecho de que la selección de los textos estaba preestablecida, según hemos señalado en la sección anterior, en las instrucciones dadas a los alumnos para este segundo periodo.

Los criterios de selección utilizados por los alumnos en la primera etapa pueden clasificarse en tres grupos. El más numeroso tiene que ver con las dificultades experimentadas en el aprendizaje de ciertos aspectos de la pronunciación del inglés, entre los que se encuentran la correcta articulación de ciertas vocales y consonantes, la identificación de grupos semánticos en la cadena hablada, la pronunciación de palabras con ciertos sufijos, la identificación y utilización de ciertas reglas de acentuación de palabras, la lectura de frases excesivamente largas, la fijación de pausas en la lectura, el establecimiento de conexiones entre palabras para leer de forma fluida, y las subidas y bajadas de distintas pautas de entonación. El segundo tipo de criterios se refiere a la comprobación por parte de los propios alumnos de su evolución a la hora de pronunciar ciertos finales de palabra y de frase, leer directamente desde trascripciones, conectar el discurso y entonar determinada forma, o bien al evaluar el funcionamiento de una regla inventada para dar cuenta de un número determinado de excepciones. Finalmente, habría un tercer grupo de criterios, bastante más variado, en el que los alumnos aluden a la necesidad de practicar determinados sonidos fricativos y africados, al gusto por un determinado tipo de texto como uno de los motivos que puede llevar a su selección, o al hecho de que el trabajo en voz alta en casa sobre los textos leídos en clase con anterioridad les ayudara a aliviar la tensión que pudieran haber sentido al leerlos frente a los compañeros y el profesor.

Aunque menor en número, el tipo de criterios en la segunda entrega es, sin embargo, más variado en cuanto al tipo de razones aducidas. Algunas de estas razones, en efecto, no aparecen citadas en los portafolios de la primera entrega, como es el caso, por ejemplo, de lo divertido que pueda ser el texto seleccionado, la riqueza en texturas tonales que pueda presentar, la variedad de velocidades de articulación utilizadas por el narrador nativo que sirve de modelo, el atractivo de los dibujos que acompañan al texto, o la importancia de poder ser capaces de identificar las diferencias entre la narración de cuentos en inglés y en español de cara al futuro ejercicio de la profesión. Todo ello parece indicar que, según avanzaban en el dominio de la pronunciación del inglés, algunos alumnos no se limitaron a utilizar criterios de selección meramente fonéticos.

\subsection{La autoevaluación}

Los aspectos relativos a la auto-evaluación suponen un giro de ciento ochenta grados con relación a las pautas que acabamos de describir. En efecto, del total de 45 
portafolios entregados en cada una de las dos etapas, los alumnos sólo aparecen evaluando sus lecturas en 11 casos en la primera fase, frente a 33 en la segunda. Además, en sólo 6 de esos primeros 11 casos llevan a cabo un análisis multidimensional (segmental y supra-segmental), tarea que, sin embargo, llegan a completar en 26 portafolios de la segunda entrega. Las cifras son, por tanto, bastante concluyentes y muestran que la decisión del profesor de hacer de la auto-evaluación una actividad obligatoria en la elaboración del portafolios durante el segundo periodo, dio sus frutos tanto desde el punto de vista de la cantidad como de la calidad de los aspectos fonéticos sometidos a análisis crítico por parte de los alumnos.

Las auto-evaluaciones de tipo segmental versaron principalmente sobre aspectos tales como la pronunciación de grupos de consonantes en posición inicial y final, la implosión de oclusivas en posición intermedia, los problemas causados por la pronunciación de la schwa o la /ae/, la correcta articulación de ciertas fricativas y africadas, o las terminaciones de ciertas palabras según los sufijos que contienen. Frente a ello, en las auto-evaluaciones de tipo multidimensional, además de los aspectos anteriores, se tuvieron en cuenta otros de carácter más discursivo como, por ejemplo, la incorrecta acentuación de formas débiles o la falta de acentuación de formas fuertes, lo ajustado de las uniones entre sonidos consonánticos entre sí y entre consonante y vocal, la corrección en la pronunciación de ciertos fenómenos de asimilación, la preocupación por no realizar una lectura excesivamente monótona en cuanto a la entonación, la identificación de efectos de "pregnancia" de unos sonidos a otros en palabras próximas de la cadena hablada, o la indebida realización de pausas y pautas de entonación.

En unos pocos casos, sin embargo, la auto-evaluación de los alumnos quedó reducida a comentarios generales del tipo "observo que aún tengo que mejorar", "pienso que he mejorado, pero tengo que seguir esforzándome" o "no domino todavía las consonantes".

\subsection{La percepción de los alumnos sobre la elaboración de los portafolios}

A los alumnos se les pasó un cuestionario de preguntas abiertas en los que se les pedía su opinión sobre las dificultades encontradas en la construcción del portafolios, los elementos que les facilitaron la labor, la percepción de su utilidad, y las mejoras que cabría introducir.

Con relación a las dificultades, los estudiantes indicaron que, en la mayor parte de los casos, les llevó cierto tiempo poder llegar a comprender el objetivo real de todo el proyecto. Les resultaba difícil entender el concepto de portafolios como algo distinto de una mera acumulación de trabajos y, por otra parte, no sabían cómo hacer visibles sus propios procesos de autorreflexión. De hecho, muchos de ellos manifestaron que necesitaban desarrollar nuevas habilidades que les permitieran tomar decisiones sobre la selección de evidencias y sobre los criterios desde los que debían presentar y evaluar sus contenidos. Sin embargo, una vez que se hicieron cargo de que debían asociar sus propios procesos de reflexión a los procesos de selección de evidencias, 
empezaron a percibir la elaboración del portafolios como un proyecto progresivamente más viable.

En esta paulatina clarificación de objetivos hubo una serie de elementos que los estudiantes juzgaron esenciales a la hora de adquirir seguridad y darle un cierto sentido a su labor: el conocer de antemano la estructura del portafolios así como los criterios de evaluación que iban a utilizarse, el asesoramiento continuo por parte del profesor, el diálogo reflexivo con los compañeros, y el grado de compromiso que llegaron a adquirir.

Con relación a la posible utilidad del portafolios como instrumento de aprendizaje, los alumnos, en un primer momento y casi con carácter general, reaccionaron de forma negativa debido a la inseguridad que les producía el tener que enfrentarse a una tarea desconocida que, en principio, parecía exigir más dedicación y esfuerzo que las tareas realizadas hasta ese momento en la Universidad. Se pasó a continuación a una segunda etapa en la que predominó la motivación, al comprobar que con el portafolios se generaba una dinámica de implicación y comunicación continua entre el profesor y ellos mismos. Finalmente, el escepticismo dio paso a la aprobación y la mayoría llegó a mostrar más confianza en que el portafolios constituía de hecho un medio muy válido para el desarrollo de las competencias relativas a la pronunciación del inglés. En estos tres momentos, sin embargo, los alumnos no dejaron de expresar sus quejas sobre la gran cantidad de tiempo que la construcción del portafolios les suponía, en detrimento del tiempo que podían dedicar a otras asignaturas. Conviene señalar que resultados similares a éstos se han hallado en contextos tan diferentes como Holanda (Smith y Tillema, 2003) o Granada (Pozo y García, 2006).

Los alumnos hicieron, finalmente, un par de recomendaciones relacionadas con la utilización del portafolios como recurso docente que suponen un verdadero cuestionamiento de la metodología docente hoy imperante en nuestras aulas. En primer lugar, sugirieron que la construcción del portafolios debería ir precedida de una etapa de formación y de información sobre las demandas implicadas, los criterios de evaluación a emplear y los beneficios a obtener de su uso, a fin de reducir al mínimo la ansiedad que puede producir el tener que enfrentarse a una situación académica nueva. En segundo lugar, señalaron que no tendría mucho sentido reducir la utilización del portafolios a una sola asignatura, ya que, de lo contrario, se podría correr el riesgo de que su implantación no estuviese coordinada entre todos los profesores del curso -como había sido el caso con el portafolios de Fonética. Ello, se argüía, podría dar lugar a un exceso de demandas académicas imposibles de atender de forma apropiada.

\section{CONCLUSIONES}

A modo de conclusión sobre los aspectos que hemos discutido en los apartados anteriores, presentamos una serie de puntos básicos que creemos pueden servir de orientación al profesor universitario para llevar a cabo una aplicación adecuada del portafolios: 
1. Hay que evitar que el portafolios sea percibido por los alumnos como un "añadido" a los objetivos y a la metodología del curso. De lo contrario, se resistirán (de forma activa o pasiva) a su elaboración, ya que lo verán como un trabajo extra que exige tiempo en una agenda ya de por sí muy recargada. Es importante hacerles ver, por tanto, que se trata de un requisito más del curso y que, como tal, va a tener su reflejo porcentual en la nota final.

2. Por ello, es muy importante delimitar, en la medida de lo posible, qué tipo de evidencias han de mostrar los alumnos y cómo las han de evaluar.

3. Con todo, el profesor ha de asumir que el alumno se sentirá perdido, sobre todo en los estadios iniciales. De ahí la importancia de la labor de orientación en esta etapa: cuanto mayor sea el trabajo inicial, menores serán, seguramente, los imprevistos que puedan surgir.

4. Se deben establecer normas claras de elaboración y funcionamiento del portafolios, tales como señalar los días de revisión conjunta, fijar los apartados optativos y obligatorios, distinguir los trabajos en grupo de aquellos que han de tener un carácter individual, etc.

5. Hay que tener buen cuidado de que las evidencias recopiladas y seleccionadas por los estudiantes se correspondan con las capacidades o competencias que éstos hayan de desarrollar. De ahí la necesidad de insistir en su diversificación cuando sea pertinente.

6. Conviene indicar a los alumnos la conveniencia llevar a cabo la selección de una o dos evidencias cada semana, de forma que adquieran el hábito de hacerlo de forma continuada y no se produzcan acumulaciones engorrosas de última hora.

7. El profesor debe sopesar con suficiente antelación el/los procedimiento(s) de evaluación que empleará, de forma que pueda darlos a conocer a los alumnos con la suficiente antelación.

\section{REFERENCIAS BIBLIOGRÁFICAS}

AGRA, M. J., GEWERC, A. y MONTERO, L. (2003). "El portafolios como herramienta de análisis de experiencias de formación on-line y presenciales". Enseñanza, 21, 101-114.

ARTEAGA BRAVO, A. y FERNÁNDEZ DEL VALLE, J. (2000). "La evaluación convencional frente a los nuevos modelos de evaluación auténtica". Psicothema, 12, 95-99.

BARBERÁ, E. (2005). "La evaluación de competencias complejas: la práctica del portafolio". Educere: La Revista Venezolana de Educación, 31, 487-503.

BERMEJO BRAVO, F. (2006). "El portafolio del alumno como estrategia formativa y de evaluación en la enseñanza superior". Jornadas Nacionales de Intercambio de Experiencias Piloto de Implantación de Metodologías ECTS. Badajoz, Septiembre.

BORDAS I. y CABRERA F. (2001). "Estrategias de evaluación de los aprendizajes entrados en el proceso". Revista española de pedagogía, 218, 25-48. 
BROWN, S. y GLASNER, A. (Edit.) (2003). Evaluar la Universidad, problemas y nuevos enfoques. Madrid: Narcea.

FERNÁNDEZ MARCH, A. (2004). "El portafolio docente como estrategia formativa y de desarrollo profesional". Educar, 33, 127-142.

Universidad Miguel Hernández, Elche, 2006. "El portafolios del estudiante" [en línea] http://www.recursoseees.uji.es/fichas/fm4.

KLENOWSKI, V. (2005). Desarrollo de portafolios para el aprendizaje y la evaluación. Madrid: Narcea.

LYONS, N. (Edit.) (1998). El uso de portafolios. Propuestas para un nuevo profesionalismo docente. Buenos Aires: Amorrortu Editores.

POZO LLORENTE, M. T. \& GARCÍA LUPIÓN, B. (2006). "El portafolios del alumnado: una investigación-acción en el aula universitaria". Revista de Educación, 341, 737-756.

SCHÖN, D. (1992). La formación de profesionales reflexivos. Hacia un nuevo diseño de la enseñanza y el aprendizaje en las profesiones. Barcelona: Paidós.

SHARP, J. E. (1997). "Using portfolios in the classroom". Frontiers in Education Conference, 1997. 27th Annual Conference. 'Teaching and Learning in an Era of Change'. Proceedings (descargado de Internet, 2-5-07).

SCHULMAN, L. (1999). "Portafolios del docente: una actividad teórica". En Lyons, N. (1999). El uso del portafolio. Propuestas para un nuevo profesionalismo docente. Buenos Aires: Amorrortu, 45-62.

SMITH, K. y TILLEMA, H. (2003). "Clarifying different types of portfolio use". Assessment and Evaluation in Higher Education, 28, 625-648.

WOLF, K. y DIETZ, M. (1998). "Teaching portfolios: purposes and possibilities". Teacher Education Quarterly, Winter, 9-22.

WRIGHT, A. W., KNIGHT, P. T. y POMERLEAU, N. (1999). "Portfolio people: Teaching and learning dossiers and innovation in higher education". Innovative Higher Education, 24, 89-103. 